\title{
Philosophiques
}

\section{Réflexion et responsabilités sociales}

\section{René Pellerin}

Volume 5, numéro 2, octobre 1978

URI : https://id.erudit.org/iderudit/203106ar

DOI : https://doi.org/10.7202/203106ar

Aller au sommaire du numéro

Éditeur(s)

Société de philosophie du Québec

ISSN

0316-2923 (imprimé)

1492-1391 (numérique)

Découvrir la revue

Citer ce document

Pellerin, R. (1978). Réflexion et responsabilités sociales. Philosophiques, 5(2), 397-416. https://doi.org/10.7202/203106ar d'utilisation que vous pouvez consulter en ligne.

https://apropos.erudit.org/fr/usagers/politique-dutilisation/ 


\title{
2. RÉFLEXION ET RESPONSABILITÉS SOCIALES
}

\author{
par René Pellerin
}

L'insistance des professeurs de philosophie, en particulier au Québec, dans la période post-thomiste et celle de l'humanisme qui semble caractériser la philosophie institutionnelle au niveau collégial depuis la fondation des Cegeps, cette insistance va dans le sens de la réflexion critique. Le cours d'introduction à la séquence des quatre cours obligatoires du cours collégial avait pour titre en 1972: La Pensée et la Réflexion. En 1974, on parlait, dans l'annuaire de la DGEC, de "réflexion radicale» et de «distanciation/appropriation" devant lesquelles il fallait faire, après les avoir réalisées, un « retour réflexif ». En 1977, on précise que l'étudiant doit non seulement savoir ce qu'est « la réflexion critique » mais apprendre à l'appliquer à son vécu, et, pour ce faire, il doit pouvoir " maîtriser les instruments théoriques et pratiques de la réflexion ". Dans toutes ces formulations, on tient compte d'une imprécision du terme «réflexion " et on spécifie de quelles sortes de réflexion on veut faire la promotion chez les étudiants.

Qu'elle soit critique, radicale, introspective ou distanciatrice, la réflexion est présentée comme un «objectif». En 1972 , la réflexion est liée à des opérations strictement internes comme «me demander, m'interroger, me rendre conscient, m'examiner ». En 1977, on y adjoint une " conséquence qui doit être l'engagement et l'action » et on ajoute un comportement: éviter les manipulations pour enfin « relativiser sa propre situation historique ». Il me semble qu'entre ces deux séries de propositions, pendant ces cinq ans, on est passé d'une préoccupation intériorisante et individualiste à une préoccupation extériorisante et collectiviste. Étant donné qu'en général les objectifs proposés ont un pas en avance sur ce qui se fait, on 
peut supposer, et on vérifie assez régulièrement, que les professeurs de philosophie n'en sont pas encore à la réalisation concrète de cette transition. Une des raisons de ce retard me semble être perceptible dans la conception imprécise de la nature et du rôle de l'activité réflexive.

Le nouveau cahier de la Digec innove de façon importante en parlant "d'instruments théoriques et pratiques » de réflexion. Auparavant, et je pense également dans le public étudiant d'aujourd'hui, on ne croit pas utile de spécifier la démarche réflexive par ses « instruments ". C'est qu'on assume le caractère évident et apodictique de la réflexion : tout le monde sait ce qu'est la réflexion et comment réfléchir. Il suffit dès lors d'intervenir ou de prendre l'initiative pour que la réflexion s'inaugure comme telle, sans distorsion et sans confusion avec ce qu'elle n'est pas. Mais l'observation et l'expérience de la discussion, avec les étudiants en particulier, sont loin de confirmer une telle connaissance que l'on prétend avoir sur ce qu'est et sur ce que doit fournir la réflexion. S'il s'agit d'engagement et d'action, il reste encore à les justifier en montrant ou en invitant au moins à voir le lien entre l'intérêt personnel et l'intérêt collectif d'un engagement exigé par la réflexion.

L’idée générale que je voudrais développer est que la réflexion est discours sélectif, c'est-à-dire qu'elle implique des choix, des valeurs et des responsabilités. Je voudrais, dans un premier temps, tenter de décrire deux falsifications réductrices de la réflexion; la réduction psychologique et la réduction «physiologique». Dans un deuxième temps, je ferai la critique d'une fonction idéologique de la promotion de l'activité réflexive: la fonction conservatrice pacifiante, et ensuite je tenterai d'élaborer l'hypothèse d'une fonction stratégique à la fois intellectuelle et sociale.

\section{LA RÉDUCTION PSYCHOLOGIQUE}

De nombreuses incitations à la réflexion se présentent dans des situations de tension entre des individus ou des groupes qui ne s'entendent pas ou, plus précisément, défendent des positions incompatibles. Dans la mesure où de telles 
situations impliquent une certaine accélération des interventions et des discours polémiques, il faut un ralentissement si l'on veut rendre la discussion "efficace". C'est sous l'idée de la réflexion qu'on tentera de favoriser ce ralentissement. Réfléchir devient synonyme de faire attention, éviter la précipitation, affirmer "prudemment ", calmement, ou tout au moins implique nécessairement alors un ralentissement dans le comportement et le dialogue. Réfléchir signifie alors prendre son temps. La traditionnelle «minute de réflexion» est une minute de pose, une suspension des activités qui brise le rythme effervescent des activités courantes, c'est le cas de le dire. À ce ralentissement se trouvent associés le calme et la modération, versant psychologique et versant moral de la réflexion, devenue attitude d'équilibre psychologique et moral. Ceux qui définissent la réflexion en disant qu'elle consiste à «s'arrêter» la définissent comme méthode psychologique tout autant que justifiée par un résultat psychologique. Si réfléchir c'est se calmer, réfléchir c'est prendre un calmant ! La réduction de la réflexion à un facteur de ralentissement et de détente est cependant la réduction psychologique la moins importante, bien qu'elle soit sans doute très fréquente. L'autre réduction psychologique qui me semble plus fondamentale consiste à réduire la réflexion à une opération de l'imagination.

Ce qu'on pourrait appeler «l'introspection contemplative " joue, en effer, souvent le rôle de la réflexion et se confond avec elle. Être impressionné et se mettre à rêver ou à imaginer des situations, des personnages, des objets, est souvent confondu avec l'activité réflexive. On utilise alors des images plutôt que des concepts ; on regarde et contemple (de façon masturbatoire ou auto-justifiante) des séquences d'un cinéma intérieur, sans intervention de la raison et d'aucune analyse théorique, et on dit être en train de réfléchir. Il en est de même pour les réactions émotives d'inquiétudes ou même d'angoisses qui sont confondues, par exemple chez certains existentialistes, avec la réflexion. Le romantisme de la méditation tranquillisante et l'idéologie de la «vie intérieure» succombent presque systématiquement à cette réduction. Si on ne précise pas le sens du mot «intérieur », on peut en effet facilement confondre ce qui se passe dans l'imagination avec ce 
qui s'appelle réflexion ou raisonnement. Réfléchir signifie alors rêver et, nonobstant la valeur psychologique du rêve, il y a là une réduction indue. Certaines personnes après avoir résisté à des pressions culturelles, sociales ou psychologiques disent qu'elles ont "besoin de réfléchir », et elles veulent clairement dire par là qu'elles ont besoin de repos, de calme, de distance et de gratifications imaginatives qui leur permettent de retrouver des forces, comme dans le sommeil. La réflexion est alors, en effet, une fuite du monde réel et agressant, un refus de la relation épuisante à la réalité et une tentative de récupération psychologique qui joue le même rôle que le rêve éveillé et volontaire : l'imaginaire. En bref, on pourrait dire que la réduction psychologique de la réflexion consiste à en faire un mécanisme de défense contre l'agression de la réalité sur la conscience et l'affectivité. L'expression "laisse-moi réfléchir "semble être une demande de paix, et l'exigence d'un respect de la personne prend la voie d'un respect du « droit » à l'introversion vers l'imaginaire.

\section{LA RÉDUCTION PHYSIOLOGIQUE}

Le miroir réfléchit une image. De cette dimension physique de la réflexion "lumineuse", on peut déduire l'origine de la réduction dont je veux maintenant parler. Si on ne conçoit pas la réflexion comme une fuite dans l'imaginaire, on peut encore toutefois la réduire en la concevant comme un reflet produit par une sorte de relation physiologique au monde. Regarder, contempler, admirer, et enfin reproduire à «l'intérieur" (dans l'imagination encore) ce qu'on a regardé, contemplé, admiré. Il s'agit ainsi, dans cette fausse réflexion, d'une reproduction en quelque sorte littérale, d'un recopiage. $\mathrm{Si}$ on peut encore parler de réduction de la réflexion à l'imagination, c'est en un autre sens que psychologique. Il s'agit ici de faire valoir la réalité et notre rapport à elle par la fidélité reproductrice. La visée de cette fidélité est de ne rien changer, et la réflexion devient «photographique ", n'exigeant du sujet que des qualités physiologiques de mémoire et de vision. L'idée d'une relation à la réalité ne suffit donc pas pour définir la réflexion, puisqu'elle laisse la possibilité de réduire cette relation à un contact physiologique et de réduire 
l'activité réflexive à une activité reproductrice d'images et d'apparences. Réfléchir devient alors synonyme de photographier, sans qu'il soit possible de vraiment réussir cette non-ingérence du sujet dans sa photo. Lorsqu'un avocat demande à un témoin qui ne se souvient plus de l'événement dont il a été pourtant témoin, lorsqu'il lui demande donc de "réfléchir ", il lui demande de reproduire dans son imagination ce qu'il a vu. En fait, il lui demande une opération physiologique intérieure, non pas une "réflexion ", sinon au sens du miroir qui réfléchit. On pourrait parler ici d'opération psychologique, mais dans la mesure où c'est un contact physiologique, voir, entendre, goûter, etc., qu'il faut reproduire, il faut tenir compte de cette relation dans l'opération mémorisante. Se rappeler, c'est un peu faire tourner un film à l'envers (et en interférant «subjectivement» ou culturellement dans cette re-production).

Un autre aspect de la réduction physiologique est la «concentration». Réfléchir, c'est se concentrer. C'est en d'autres termes se mettre au focus, si on veut prendre encore l'exemple de la photographie. La concentration est dans la même position sémantique que la réflexion lumineuse. Ce terme indique une densité, et dans le langage ordinaire il indique une haute densicé. L'eau de Javel concentrée contient plus du produit que l'eau de Javel diluée. Il y a donc là encore un aspect physique, presque un aspect quantitatif. Réfléchir devient alors une opération de haute densité, comme si nos yeux ou notre mémoire "travaillaient » plus en se focalisant sur un seul point de la réalité. On conçoit alors la réflexion comme s'il s'agissait d'une opération physiologique de l'œil qui essaie de ne regarder qu'un point à travers un brouillard de particules. La réflexion se trouve réduite à un aspect du travail du corps ou des sens (système nerveux). Si le témoin de notre avocat ne réussit pas à se rappeler l'événement, l'avocat insistera en demandant de «bien réfléchir », c'est-à-dire de réfléchir «plus», c'est-à-dire encore de «voir mieux» en travaillant plus fort avec son système sensoriel et sa mémoire.

\section{LA FONCTION CONSERVATRICE PACIFIANTE}

Toutes ces réductions ne sont pas des erreurs, mais elles 
sont des confusions entre des aspects secondaires ou partiels et les aspects essentiels, qui ne sont pas mentionnés ou dont on ne tient aucun compte. En associant réflexion avec lenteur, calme, reproduction littérale (symétrique) et introspection contemplative, on fait valoir sans le dire une fonction de la réflexion dont il faut maintenant faire état. Il est fréquent qu'on se fasse demander de réfléchir au moment où l'on se montre en désaccord avec quelqu'un. Comme si réfléchir c'était parvenir à un point précis de connaissance ou de vérité déjà établi, un peu à la manière du souvenir qui doit être fidèle à une réalité extérieure et "objective ". La réflexion dans cette situation a clairement une fonction sociale, celle de conduire quelqu'un à admettre et non à trouver quelque chose d'encore absent. Tout se passe comme si on disait : "si quelqu'un réfléchit bien, il verra clairement que ceci est comme cela". Cette attitude comporte une définition fonctionnelle ou falsifiée au nom de l'efficacité pratique, laquelle répond à son tour à une intention inavouée. Il en est de même de ceux qui incitent à la réflexion d'autres personnes qui leur semblent trop énergiques ou trop "rapides». Sous le couvert de la réflexion, c'est en fait une attitude plus modérée qui est demandée. Là encore la réflexion sert de prétexte à une exigence sociale : le respect des autres étant confondu avec le laisser-faire et la passivité « réflexive». Le retour sur soi exigé dans et par la réflexion constitue également un renoncement (même provisoire) à l'action. Dans cette mesure, ce retour peut servir davantage à suspendre l'action qu'à l'orienter ou qu'à la préparer. En invitant quelqu'un à réfléchir, je peux essayer de m'en débarrasser en le retournant ou le faisant se retourner sur lui-même. En disant que l'on ne peut jamais être absolument certain de la «valeur de nos valeurs", on peut vouloir paralyser l'action et l'engagement et favoriser la réflexion comme facteur de déviation et de conservatisme. Il se peut que ce retour sur soi se présente comme bénéfique, au moins éventuellement, tout autant à soi qu'aux autres; mais il se peut aussi que je sois assez habile pour faire douter celui qui veut affirmer ou veut faire quelque chose que je n'accepte pas moi-même. C'est un fait d'expérience qu'on tient plus souvent à faire réfléchir ceux qui ne partagent pas ses valeurs et ses préjugés que ceux qui les partagent. On sait aussi que celui 
qui n’a pas réfléchi, ce n'est pas nécessairement « l'autre». Celui qui s'éloigne du statu quo et des habitudes, sociales et culturelles plus particulièrement, semble fréquemment n'avoir « pas assez réfléchi », comme si réfléchir c'était « confirmer» et admettre.

En tant que frein à l'action intempestive, la réflexion sert parfois à favoriser l'efficacité de ce qui ne peut être fait de façon intempestive. Mais ce ne sont pas tous les engagements qui doivent être calmes et lents. La réflexion, en ralentissant l'action, peut devenir «folle» de telle sorte qu'elle ne pourra plus s'arrêter et sortir d'elle-même, hallucinée qu'elle sera dans le monde de la recherche pratiquement paralysante de l'absolu ou du définitif. On compte fréquemment sur le temps et l'oubli, sur l'inquiétude et le perfectionnisme latent, pour se protéger contre des interventions qui modifient trop profondément nos convictions ou notre manière d'agir. Mais on compte aussi sur une certaine manière de faire réfléchir et sur la valorisation simpliste de la réflexion comme valeur en soi, pour neutraliser les opérations sociales ou individuelles qui modifieraient l'état actuel des rapports et des comportements interpersonnels. Ceci vaut aussi bien pour la réflexion que pour la philosophie institutionnelle. Comme le disait Michel Dufour du Cegep de Maisonneuve, au congrès de la S.P.Q. en 1975, "LA PHILOSOPHIE CRITIQUE n'est pas une épée de Damoclès frisant la tête du pouvoir ; tout au plus une épingle à chapeau aiguillonnant les arrière-trains qui sommeillent". Certains perçoivent davantage "l'épée " dans la réflexion radicale ; une autre sorte de réflexion peut précisément alors, et assez paradoxalement, servir à éviter cette épée aussi bien que l'épingle. Il faudrait donc distinguer, de façon claire et non équivoque, la réflexion critique et la réflexion "méditative ". L'une sert, en effet, à ré-évaluer ce qui semble aller de soi et en ce sens elle "détranquillise ", l'autre, au contraire, sert à calmer, ralentir, voire même paralyser l'action concrète au profit d'une action "intérieure " qui peut facilement devenir "schizophrénique », le monde intérieur et imaginaire étant en général beaucoup plus agréable que la réalité vécue.

Dans la mesure où l'on n'a pas encore trouvé ce qui est à la fois universellement et fondamentalement vrai et bien, la 
réflexion, comme travail "intérieur » qui s'oriente vers un approfondissement radical, doit être menée comme si elle ne pouvait JAMAIS se terminer. On se laisse trop rarement impressionner par les conséquences d'une telle vérité de l'expérience. Et pourtant même ceux qui ont cherché avec le plus d'énergie la certitude absolue, comme Descartes, reconnaissent cette limite et en tiennent compte pour l'action qui n'attend pas la fin de nos réflexions ${ }^{1}$. Si j'attends de parvenir à la vérité universelle, pensait Descartes, pour agir, lorsque l'action sera due, il sera trop tard; et il proposait de suivre l'opinion commune. En ce sens on comprend pourquoi on peut dire qu'il faut réfléchir, sans préciser pendant combien de temps et jusqu'où, et en disant simplement qu'il faut réfléchir "suffisamment». En ce sens, on comprend aussi pourquoi réfléchir veut aussi dire, entre autres, «attendre » parce que effectivement la réflexion «fait attendre " l'action, disons « corporelle» et «publique ». Mais il faut voir qu'une telle attente laisse les choses être ce qu'elles sont et ainsi «accepte », au moins en fait, un statu quo. Si dans plusieurs sphères de la réalité, une telle acceptation est sans conséquences graves sur la vie sociale, dans d'autres cependant ces conséquences sont inacceptables. Que serait-il arrivé de la civilisation si les médecins avaient attendu de trouver la Raison fondamentale de vivre avant de travailler à guérir? Et pourtant, il faut ici tenir compte d'un magistral contre-exemple. Que serait-il arrivé si les représentants de la justice sociale, avocats et juges, n'avaient pas réfléchi à la culpabilité avant de condamner, que serait-il arrivé si ces autorités avaient réfléchi un peu plus à la fonction des prisons avant d'y envoyer des milliers de personnes? S'il est vrai que dans le doute il faut s'abstenir, il n'est pas vrai que tout doute soit justifié comme frein à l'engagement concret. Dans certains cas, il faut réfléchir et s'abstenir si la réflexion ne donne pas de fruits assez mûrs, mais, dans d'autres cas, il semble qu'il faille agir sans attendre que la réflexion parvienne à son terme. Il faut alors se fier à autre chose qu'à la réflexion qui cherche l'évidence finale et définitive. La fonction conservatrice de la réflexion est une

1. Gilles Lane, dans Lurgence du Présent, (Montréal, P.U.Q., 197.3), donne un excellent exemple de cette attitude "réaliste". Le second chapitre de la deuxième partie de son livre s'intitule : « En attendant ": perspecrive d'éducation 
fonction qui doit être citée au procès des valeurs et des priorités. Si j'attends que mes enfants me demandent de les éduquer à la vie sociale, il sera trop tard. Mais si je condamne avant de réfléchir sur les conséquences et les justifications de ma condamnation, je serai source d'injustice. Le romancier Samuel Becket, préoccupé par le problème de l'incomplétude irréductible de toute œuvre romanesque qui ne peut révéler toute la vérité d'un personnage ni même y accéder, voyait ce problème avec un désespoir que l'on comprend, même si on ne le partage pas :

«. . mais il faut choisir entre les choses qui ne valent pas la peine d'être dites, et celles qui le valent encore moins. Car si on voulait tout dire on n'en finirait jamais et tout est là, finir, en finir ${ }^{2}$."

Si l'on ne veut pas en finir on se "libère ", mais c'est pour se perdre dans le désert du discours sans fin qui ne touche à rien et ainsi conserve des situations avec toutes leurs indécidables mais réelles injustices.

\section{FONCTION STRATÉGIQUE INTELLECTUELLE ET SOCIALE}

Si la réflexion est discours, elle n'est pas n'importe quel discours. Ce discours peut être lent ou rapide, calme ou énergique, auto-critique ou altéro-critique, stimulant ou stérile, stratégique ou esthétique. Il est toujours cependant le résultat d'une relation interrogative, soit avec les événements, soit avec les personnes. Il est également toujours inachevé et n'a pas de valeur en lui-même. Il vaut par ses contenus, ses objectifs et ses résultats. Il est discours s'il est articulé et progressif, soit de façon linéaire soit de façon concentrique, soit simplement de façon exploratoire. Ce sur quoi je veux ici m'attarder c'est la fonction stratégique de ce discours.

\section{a) Stratégie intellectuelle}

Le discours de réflexion est une interrogation qui se cherche non seulement un aboutissement dans une réponse, mais aussi et surtout un départ dans une question, pertinente à un objectif. Le premier objectif semble être celui d'une

2. Samuel Becket, Molloy, Paris, Union Générale d'éditions, 1963, «10/18 », p. 53. 
connaissance à acquérir ou à clarifier. Il faut donc localiser ce qu'il faut connaître et sous quel angle le connaître. C'est la recherche d'une définition, d'une précision, d'une explication qui prend d'abord la priorité. La réflexion se présente ainsi comme une réaction à une perception qui fait problème pour l'intelligence. À partir de cette perception qui fait impression et qui focalise l'attention sur un domaine de l'expérience, l'individu cherche la question à poser. Il réfléchit. Il cherche à comprendre et à s'accaparer, disons "intellectuellement", ce qui s'offre et s'impose à lui. En ce sens la réflexion exige une première relation d'expérience avec la réalité, mais ne se réduit pas à cette relation. Elle exige un dépassement de la factualité au profit d'une intelligibilité, soit du sens, soit du fonctionnement, soit de la situation de ce qui est à intelliger.

En tant que stratégie intellectuelle, la réflexion est un détour. Elle cherche à voir $\mathrm{CE}$ qu'il faut savoir, et elle consiste en une recherche de ce savoir. La distance qui s'établit alors entre le sujet connaissant et l'objet à connaître est condition d'une activité de "rupture physique », et en même temps d'une nouvelle relation, disons de conceptualisation (en partie linguistique). La réflexion sert ainsi à briser le contact " corps à corps " pour établir un contact par «traduction », du senti à la formalisation. C'est authentiquement un travail qui s'effectue dans cette traduction, puisqu'il faut alors parler de ce qui est d'abord choisi comme révélateur et significatif, puis mettre en relation ces éléments d'explication. Il faut construire une description sélective et fonctionnelle, une structure qui « rende compte » des apparences et des comportements de l'objet ou de la réalité à laquelle on réfléchit. La réflexion consiste à faire durer la traduction formalisante et explicative pour épuiser le plus possible ce qu'il faut expliquer et formaliser. En ce sens, elle est tout autre que l'observation contemplative. Elle est mancuvre et travail. Elle construit.

Cette construction est aussi une destruction du premier contact et des résultats immédiats du premier contact. Elle est affrontement d'un sujet avec ses propres perceptions et ses propres évidences non critiques. C'est son aspect « individualiste " et c'est, en général, cet aspect qui est retenu dans l'incitation à la réflexion calmante et ralentissante ou contem- 
plative. Le sujet qui réfléchit établit ses propres valeurs et ses propres évidences en accusées et opère ainsi sur lui-même un renversement critique. Il doute de lui-même et, d'une certaine manière, entreprend une autodestruction dont il espère sortir vainqueur. Il perd ses «illusions" et peut aboutir à perdre ainsi tout ce dont il n'est pas absolument certain et/ou que les autres n'approuvent pas. On voit comment il peut être «machiavélique " de faire réfléchir dans cette perspective. On suppose alors que le rêve d'une action parfaitement juste et fondée sur une vérité absolue peut être réalisé avec un peu, beaucoup, de patience et de "réflexion », et ainsi on mise sur la faiblesse «théorique », pour ainsi dire, des justifications de l'engagement critique et réformateur. Si donc la réflexion est destruction, c'est qu'elle assume la non-absoluité de l'évidence première. Ne concluons pas de cela qu'aucune vérité n'est possible, mais concluons simplement que la vérité humaine ne peut être qu'humaine, c'est-à-dire qu'elle est à reprendre comme jamais définitivement «donnée » ou «possédée », d'une part, et, d'autre part, inévitablement dépendante de la situation de l'homme comme membre responsable dans et pour une collectivité. La réflexion détruit l'isolement en l'assumant comme départ vers un "rapprochement».

La construction et la destruction qu'est la réflexion ne peut être que personnelle, c'est-à-dire marquée de l'originalité et des projets d'une conscience. Elle doit se distinguer radicalement de tout ce qui n'est pas elle et de tout ce qui n'est pas d'elle. Non pas qu'elle soit créatrice, mais, en tant que retour sur la perception et l'affirmation, elle implique « distanciation». Celle-ci met le sujet qui réfléchit en «évidence », elle le met en présence distante et interrogative. Réfléchir, c'est s'isoler par l'instauration d'une relation « invisible » entre soi et soi, ou entre soi et les réels ou les autres qui parlent de ces réels. Mais c'est aussi s'affirmer comme résistance et intériorité imprenable. Par là la réflexion devient question plus que réponse, elle devient stratégie du doute qui souvent constitue un affront pour celui de qui on doute. Ce sera son aspect social.

La réflexion, en tant que stratégie intellectuelle, n'est pas une «méthode» de connaissance, mais elle constitue plutôt 
une attitude. Peut-être vaudrait-il mieux parler toujours d'activités réflexives plutôt que d'une sorte de réalité autonome que serait LA réflexion. Le mouvement linguistique qu'est le questionnement pourrait mieux en effet définir le dynamisme de l'activité réflexive. Il faudrait alors y adjoindre l'interrogation persistante et la mise en doute des évidences communes ou méconnues. Dans la réflexion le sujet va plus loin. Il laisse derrière lui quelque chose, le suspend, l'interroge en accusation, soit de manière optimiste (vrai jusqu'à preuve du contraire), soit de manière pessimiste (vrai seulement si preuves suffisantes). Par ces manières d'interroger, la réflexion est marquée par la personnalité et l'expérience du sujet, et ainsi on ne peut définir une seule manière de réfléchir qui serait LA vraie réflexion. Rappelons, par exemple, que le Cogito chez Descartes est né d'une expérience d'insatisfaction du milieu intellectuel de son époque. De même que la critique nietzschéenne est née d'une expérience semblable.

Il ne faut pas cependant s'en tenir à cette dimension négative de la réflexion. Une ambition est à l'ouvre dans l'activité réflexive. Chez Descartes et chez Kant, elle est intellectuelle et pratique. Elle est également intellectuelle et pratique chez Marx et Nietzsche. Dans sa dimension de stratégie intellectuelle, la réflexion est exigence de compréhension et, à ce titre, elle exclut la simple introspection contemplative ou la médiation calmante. Elle implique, par ailleurs, une méthode et un travail effectué selon cette méthode. Il n'y a pas de réflexion sans travail du langage interrogateur et explorateur de formulations, d'hypothèses, de dimensions cachées, de sens et de finalités explicites ou implicites (en particulier : idéologiques), de cohérence et d'intelligibilité. Une affirmation n'est signe de réflexion que si elle est stratégie vers une meilleure compréhension. Autrement, elle est autodéfense et stratégie psychologique ou sociale, mais non stratégie intellectuelle. Encore que la réflexion puisse être stratégie sociale orientée vers une compréhension "collective", comme nous le verrons plus loin. Rappelons simplement ici que si la réflexion est personnelle au sens indiqué plus haut, elle n'est pas « individuelle», bien au contraire. 
Il ne faudrait pas croire que la dimension opérationnelle de la réflexion, en tant que construction-destruction et travail interrogateur, implique un point de départ «imaginaire» hors de l'existence concrète comme s'il s'agissait d'un axiome ou d'un postulat purement formel. La réflexion est dépendante d'une "intuition maternelle ». À partir d'une expérience de la réalité observée ou « vécue », le sujet réagit par une interrogation qui s'appuie sur une ambition d'abord intellectuelle. Mais il faut reconnaître que cette ambition suppose déjà une croyance, une conviction ou une expérience axiologique (une valorisation fondée dans une perception personnelle du monde et du devoir être). L'insuffisance ressentie devant la simple constatation d'un phénomène amène la nécessité d'un retour à cette conviction (et à cette ambition qui lui est liée) et par lesquelles le sujet cherche à prendre position à propos de ce phénomène et de façon adéquate. Le sujet ne réfléchit pas de sang-froid, mais en vue de la réalisation d'un objectif. Et cet objectif constitue pour lui un projet qui conditionne non seulement l'existence même de ses réflexions, mais également ses modalités de réflexion. On ne peut donc appuyer la réflexion sur des objets qui nous laissent indifférents.

La réflexion commence lorsque le sujet est provoqué par l'expérience de la réalité à propos d'une croyance déjà établie en lui. Il se fait alors l'obligation de TENIR COMPTE de ce qu'il reconnait comme admis pour comprendre ce qu'il n'admet plus ou qui se présente comme incompatible avec ce qu'il a admis. Dans la mesure où ce qui est ainsi admis comme «fondement » n'est pas consciemment reconnu, la réflexion devient «idéologique ». Dans la mesure cependant où cela est consciemment reconnu, la réflexion se reconnaît comme « dépendante " d'un irréfléchi grâce auquel la réflexion peut être entreprise comme distanciation "optimum». Cela ne signifie nullement que l'origine de la réflexion à propos d'une occasion particulière puisse être fixée arbitrairement. C'est dans l'expérience subjective immédiate qui contraint la reconnaissance d'une évidence indémontrable, mais exigeante, que s'établit le fondement de la réflexion. À la manière de Descartes, on pourrait dire qu'il est impossible de réfléchir à la valeur de la réflexion sans admettre déjà cette valeur. C'est en 
ce sens que la réflexion ne peut jamais s'achever, comme nous l'avions déjà affirmé plus haut. Cela ne veut pas dire que l'on ne puisse pas cependant établir que dans certaines situations, ou plutôt dans certains types de situations, la réflexion n'a pas de valeur, ou pas de valeur prioritaire, ou pas de valeur exclusive.

\section{b) Stratégie sociale}

Que la réflexion relève des opérations intellectuelles " intérieures ", on le reconnaîtra facilement. Qu'elle soit une activité personnelle et qui relève d'une responsabilité personnelle, on l'admettra également. Mais que la réflexion constitue une stratégie avec des implications sociales inéluctables, cela semble moins évident. Tout au plus sera-t-on porté à reconnaître cette implication pour certains sujets de réflexion ou pour certaines conséquences de la réflexion. Je voudrais montrer ici que toute réflexion, par ce qui la constitue comme travail interrogateur effectué dans "l'intériorité " du personnel, a des implications sur le social, sur les autres.

Le cahier de l'enseignement collégial de 1977 indique que la réflexion doit avoir pour conséquence l'engagement et l'action. Il y a déjà là une relation explicite entre ce qui se passe dans l'imaginaire et ce qui se passe dans le milieu concret de la collectivité. Mais il faut aller plus loin et se demander pourquoi on doit tenir compte de cette « conséquence " dans la promotion de l'activité réflexive. Si l'on réussissait à montrer l'inéluctable implication sociale de la réflexion, comme travail interrogateur, on pourrait ensuite plus facilement voir comment cette implication doit se faire consciemment dans une direction moralement positive ou justifiable pour la conscience.

L'intention de comprendre qui semblait à l'œuvre dans l'inauguration de la réflexion, intention qui s'exprimait à l'occasion d'une expérience stimulante de la réalité, doit être comprise à l'intérieur ou en fonction d'une intention plus fondamentale et plus englobante encore. Il y a certes une inadaptation qui cherche à se dissoudre grâce à la réflexion ; mais si la réflexion est un travail qui produit ou cherche à produire un «objet», elle implique une manipulation 
constante d'instruments de compréhension sur une réalité extérieure reliée à d'autres consciences que la mienne. Si je réfléchis sur la pertinence d'une stratégie politique, par exemple, je réfléchis aussi sur les personnes qui sont concernées par cette stratégie, soit parce qu'elles la défendent, soit parce qu'elles auront à en subir les conséquences humaines. Si je mets en question une décision ou une attitude, je réfléchis en me confrontant avec ceux qui prennent pour acquises cette décision ou cette attitude. Si je réfléchis sur une hypothèse concernant le « comportement » de l'électron, je travaille à une compréhension qui concerne d'autres chercheurs ou des utilisateurs possibles de cette compréhension. Si je ne communique pas le résultat d'une réflexion qui me semble prometteur, je prive des personnes d'un bénéfice dont je suis dépositaire.

Il semble que dans la plupart des cas de réflexion soutenue, il y ait une autre intention à l'œuvre que celle du seul progrès des connaissances ou de l'adaptation individuelle à la réalité. Dans lá mesure où l'on ne perçoit pas d'autre intention que celle-là, on peut considérer qu'un retrait de la réalité sociale est opéré, parfois même délibérément, et qu'il s'agit alors d'une réflexion qui est «libérée ", de façon même répréhensible, de la question des valeurs et des justifications sociales. L'autre intention que l'on espère donc à l'œuvre serait une intention moralement justifiable grâce à laquelle le retrait social, le retour à soi-même dans son propre imaginaire, pourrait être en quelque sorte "excusé » ou mieux encore pourrait être justifié malgré son apparente immoralité ou a-moralité. Une réflexion soutenue implique, en effet, une suspension de l'engagement visible dans la réalité sociale et, en ce sens, pourrait être considérée comme un signe d'égocentrisme et d'a-socialité. En privant les autres de quelque chose que je peux faire et qui serait profitable aux autres, idéalement sans me nuire, je fais acte de refus de collaboration et de participation. En ce sens, je me retire de la collectivité qui me fournit pourtant tous mes instruments et tous mes sujets de réflexion. Si cela peut être justifié, ce ne pourra être qu'au nom de valeurs supérieures à celle de la simple possession individuelle et "privée » de connaissances, et ce ne pourra être qu'en vertu d'une intention assumée de ne pas me retirer 
vraiment de ma responsabilité sociale, mais au contraire de mieux m'y engager. En ce sens, la réflexion n'est pas justifiée par la possibilité d'une connaissance, mais plus fondamentalement par son caractère éthique et social. Elle implique une obligation et une responsabilité pour être justifiée comme arrêt de l'action visible. Elle est elle-même engagement, dans des conditions idéales, en faveur d'une collaboration moralement positive pour la collectivité. Il s'agit d'un engagement à propos d'une compréhension, donc d'un choix qui doit moralement être orienté vers la collaboration sociale pour être justifiable devant la collectivité.

Celui qui réfléchit de façon soutenue veut réconcilier (comprendre) et il cherche à éviter ou à assumer un affrontement selon des facteurs de promotion humaine-sociale. La lutte contre le dogmatisme, par exemple, qui suscite et oriente la réflexion, ne peut pas se faire de façon dogmatique ou intransigeante, parce qu'alors la méthode contredirait l'objectif. Le retour à la réalité par-delà les «opinions » personnelles, ou le retour au consensus fondamental par-delà les divergences ou les oppositions idéologiques ou méthodologiques, ne doit se faire qu'au nom de et que selon la logique interne d'une valeur justifiante, surtout si ce retour implique une intervention sur les autres. Éviter l'affrontement en réfléchissant ou en faisant réfléchir exige, pour être justifié, qu'une ambition justifiable soit à l'œuvre dans cet évitement. Il peut ainsi arriver que l'affrontement permette mieux la réalisation de cette ambition que l'attitude pacifiste qui veut la paix comme besoin individuel. Laisser tomber une discussion ou renoncer à convaincre n'est pas nécessairement le signe d'un souci de la promotion de l'autre, mais peut au contraire marquer la présence d'un refus d'une responsabilité. Par ailleurs, certaines façons de convaincre ou de discuter (comme réflexion extériorisée et conduite avec une collectivité par exemple) impliquent au plan des attitudes la présence d'une ambition qui, malgré les apparences, est tout autre qu'altruiste, s'orientant plutôt vers une pseudo-victoire individuelle sur un " adversaire ».

On ne verrait pas pourquoi on laisserait quelqu'un réfléchir pendant que l'action urge et que des gens souffrent de notre "retour à soi », on ne verrait pas non plus pourquoi on 
peut forcer quelqu'un à réfléchir alors qu'il se sent en possession d'une évidence suffisante, si on ne supposait pas une intention «positive» dans l'activité réflexive. La vérité ou la connaissance ne suffisent pas à établir cette positivité dans la mesure où la recherche de la vérité implique un choix de priorités en face d'autres urgences que celle de la connaissance. Si l'on choisit la recherche de la vérité, ce ne peut être qu'en assumant une exigence qui ne sera admise par la collectivité que si elle y trouve sa place. Le problème se pose de façon assez évidente dans les subventions gouvernementales de recherches scientifiques, aussi bien d'ailleurs que dans l'instauration de cours obligatoires de formation "générale " à certains niveaux d'enseignement. Le problème se pose également pour ceux qui ne tiennent pas à communiquer leurs réflexions pour des raisons qui ne concernent pas l'efficacité d'une telle communication, mais plutôt le refus d'une responsabilité à une telle communication. On voit mal comment on pourrait justifier le salaire d'un professeur qui enseignerait parce qu'il " aime " sa matière et qu'il aime en parler, sans se soucier de collaborer à la collectivité par une communication de connaissance, et de collaborer en même temps à la promotion personnelle de ceux qui viennent l'entendre.

La réflexion amène donc la communication comme un de ses devoirs. On fait d'ailieurs l'expérience fréquente d'une meilleure qualité de réflexion lorsque l'on réfléchit avec quelqu'un. La dimension individuelle de la réflexion est ainsi plus apparente que réelle dans les cas où la réflexion est justifiée. Celui qui réfléchit est constamment en présence, réelle ou imaginée, explicite ou implicite, de la collectivité. Il utilise des méthodes d'observation, d'analyse, de déduction ou d'explication, il produit des hypothèses, des questions, des définitions en vue d'une compréhension à transmettre ou à utiliser pour une intention qui doit être admissible par ceux qui, de loin ou de près, sont concernés par ces opérations qui sont choisies et utilisées par un individu au milieu de la collectivité et pendant que cette collectivité attend. Celui qui réfléchit reçoit des approbations, des réponses, des critiques, des remarques, des compléments, des objections, des questions, et il doit répondre s'il assume la responsabilité inhérente 
à l'activité réflexive, comme il doit avoir le souci d'arriver à la communication dans un délai " raisonnable».

Si le proverbe qui veut que toute vérité ne soit pas bonne à dire a un sens, c'est parce qu'il tient compte d'une dimension supérieure à la vérité, la dimension axiologique de la promotion bumaine-sociale. Il faudrait compléter d'ailleurs ce proverbe en disant que toute vérité n'est pas bonne à chercher et que toute vérité n'est pas bonne à défendre. C'est toute la recherche d'une vérité objective, universelle et définitive aussi bien chez un individu que chez des groupes de professionnels de la "science " ou de la "philosophie ", qui doit être confrontée à la justification axiologique. C'est grâce à la liberté même de vouloir savoir que l'on peut choisir de savoir pour assumer une responsabilité plus fondamentale que celle de la raison qui veut "factuellement" comprendre et devant laquelle le sujet conscient doit prendre position au nom d'une justification admissible par la collectivité où a lieu la rationalisation.

Ce qui justifie la volonté de savoir peut aussi justifier le refus de savoir. Dans la mesure où la réflexion est travail interrogateur, il faut tenir compte du statut de ce sur quoi porte la question, spécialement si c'est sur un homme que l'on fait porter notre quête de savoir.

«En notre temps, sur notre planète, savoir un homme, c'est l'interroger jusqu'à la vivisection. Nous tous nous savons ce que peut contenir de charge sadique le mot question. L'inquisiteur envahit tout. Connaître un homme, c'est en fait ne lui laisser aucune chance d'exister. Alors je ne le questionne pas. Je le regarde, le palpe, le respire, bouleversé par la force d'inconnu qu'il me communique . . . 3 "

On peut comprendre cette forme très raffinée de respect. Mais c'est surtout l'intention et le souci moral qui justifient un tel refus de savoir. Si réfléchir, c'est refuser de conclure une fois pour toutes et assumer dans nos engagements une radicale insuffisance cognitive, spécialement lorsque cet engagement concerne la dimension personnelle, alors la réflexion pourrait non seulement ne pas s'opposer mais contribuer à assumer un

3. Ipousteguy, 1974, cité par André Glucksmann, La cuisinière et le mangeur d'bomme, Paris, Seuil, 1975 , p. 187. 
respect des autres, sans trop risquer de négliger la responsabilité de la collaboration au progrès commun dans une collectivité ou une simple relation interpersonnelle. Dans certains cas, il faudrait demeurer vigilant pour que l'absence de questions ne soit pas une manière subtile de dominer, écraser, manipuler, tromper ou mépriser et «oublier» la possibilité d'une contribution positive au projet collectif de mieux-être. Si je veux, en effet, assumer une collaboration sociale qui permette un progrès à la fois individuel et collectif par une indissoluble unité de progression, je ne peux pas toujours et dans tous les cas me satisfaire d'une attitude seulement "réceptive». Cette réceptivité demeure essentielle, mais doit être perçue comme élément d'un ensemble d'attitudes tout aussi essentielles comme celles du souci de la promotion humaine-sociale. Si, par exemple, quelqu'un se plaint d'une souffrance psychologique devant et à moi, je ne peux demeurer indifférent à la « force d'inconnu" qui le mine. Et ma façon de ne pas rester indifférent s'orientera vers un engagement concret qui répondra à une authentique intention de collaboration si je reconnais ce sens aux questions que je devrai lui poser pour connaitre ce à propos de quoi je veux l'aider. Ce à quoi il faut être attentif dès lors, c'est aux priorités qui pourraient justifier une aide pertinente pour l'individu que je veux aider au nom d'un intérêt au progrès commun. Le droit au respect de la dignité peut sérieusement remettre en question nos modes assez « naifs » d'altruisme et de générosité 4 . Par ailleurs, la collectivité reconnaît de plus en plus la possibilité pour l'homme de corriger les injustices du sort en fournissant aux défavorisés des droits qui sont justifiés par la reconnaissance de devoirs pour ceux qui sont mieux pourvus.

Ceci nous reconduit au problème de la réflexion au niveau même de la stratégie sociale. Ceux qui peuvent élaborer une réflexion et ainsi augmenter leur chance, même sans certitudes, d'une action mieux adaptée au projet fondamental de l'homme, sont des gens «pourvus» qui ont des devoirs

4. Ce "paradoxe des conséquences", dont Weber parlait au sujet des yrandes entreprises politiques, vaut aussi au plan des groupes restreints et des relations interpersonnelles. Il s'agit d'un phénomène assez peu étudié bien que très souvent perçu et qu'on nomme en sociologie : l'effet pervers. (CF. Raymond Boudon, Effets penters et ordre social, Paris, P.U.F., 1977). 
devant ceux qui sont moins pourvus. Cela ne vaut pas que pour des performances intellectuelles, mais aussi pour des capacités psychologiques ou des libertés qui ne sont pas également distribuées dans la collectivité et qui favorisent des individus plutôt que d'autres, dès lors défavorisés sur ce point déterminant. C'est grâce à cette dimension de la réflexion que l'on peut intervenir en faveur de personnes qui pourraient profiter de notre réflexion en même temps que nous. La réflexion est recherche d'un rapport à la réalité qui puisse rallier les hommes. Elle est une exigence de conscience pour celui qui choisit de donner priorité au rapprochement de tous les hommes plutôt qu'à la victoire de certains hommes, même s'ils constituent une majorité, sur d'autres hommes.

Collège Laflèche

Trois-Rivières. 